\title{
Stabilization and Synchronization of Unified Chaotic System via Impulsive Control
}

\author{
Cheng Hu and Haijun Jiang \\ College of Mathematics and System Sciences, Xinjiang University, Urumqi, Xinjiang 830046, China \\ Correspondence should be addressed to Cheng Hu; hucheng@xju.edu.cn
}

Received 22 May 2014; Accepted 5 June 2014; Published 26 June 2014

Academic Editor: Yonghui Xia

Copyright (C) 2014 C. Hu and H. Jiang. This is an open access article distributed under the Creative Commons Attribution License, which permits unrestricted use, distribution, and reproduction in any medium, provided the original work is properly cited.

\begin{abstract}
The impulsive control and synchronization of unified chaotic system are proposed. By applying impulsive control theory and introducing a piecewise continuous auxiliary function, some novel and useful conditions are provided to guarantee the globally asymptotical stabilization and synchronization of unified chaotic system under impulsive control. Compared with some previous results, our criteria are superior and less conservative. Finally, the effectiveness of theoretical results is shown through numerical simulations.
\end{abstract}

\section{Introduction}

In 1963, Lorenz presented the first chaotic attractor in a simple three-dimensional autonomous system [1]. Since then, many researchers began to study chaos theory. In 1999, Chen and Ueta found another chaotic system called Chen system, which is similar but topologically nonequivalent to Lorenz system [2]. In 2002, the unified chaotic system was introduced by Lü and Chen. It includes Lorenz system and Chen attractor as two extremes, respectively, and Lü attractor as a transition system between Lorenz system and Chen system $[3,4]$.

For a long time, people thought that chaos was neither predictable nor controllable. However, in the 1990s, the viewpoint and theory about control of chaotic system introduced by Ott. Grebogi Yorke and Pecora Carroll completely changed the situation [5], and many scholars began to study chaos control. Until now, many different techniques and methods have been proposed to achieve chaos control and synchronization, such as adaptive control [6], fuzzy control [7], and impulsive control [1, 5, 8-22].

In the past several years, impulsive control strategy has been widely used to stabilize and synchronize nonlinear dynamical systems. From the control point of view, impulsive control is an effective method based on the theory of impulsive dynamic systems [14, 17, 23]. It allows stabilization of a complex system by using only small control impulses. Additionally, this method drastically reduces the amount of information transmitted from the driving system to the driven system in chaos synchronization $[10,20]$. Those merits make it more efficient and more useful in a great number of real-life applications. Up to now, the stabilization and synchronization of chaotic systems under impulsive control have been extensively studied (see $[1,5,8-10,15,16,19-22]$ ).

In this paper, based on impulsive control theory, the stabilization and synchronization of unified chaotic system are investigated via designing impulsive controller. By introducing a piecewise continuous function, some new and useful criteria are established to guarantee the stabilization and synchronization of unified chaotic system. Compared with the main criteria obtained in $[8,10]$, our results are less stringent and more general.

This paper is organized as follows. In Section 2, two central lemmas are given. In Section 3, the impulsive control model of unified chaotic system is introduced and some new conditions are provided to ensure the stability of the equilibrium point for unified chaotic system under impulsive control. In Section 4, impulsive synchronization criteria are proposed. In Section 5, numerical simulations are provided to illustrate and verify our results.

\section{Preliminaries}

In this section, two central lemmas are provided to support our analysis in later sections. 
Lemma 1. Assume that $A$ is an $n \times n$ symmetric and semipositive definite real matrix; then, for any two real column vectors $\zeta \in R^{n}$ and $\eta \in R^{n}$, the following inequality holds:

$$
\left|\zeta^{T} A \eta\right|^{2} \leq\left|\zeta^{T} A \zeta\right| \cdot\left|\eta^{T} A \eta\right|
$$

Proof. Since matrix $A$ is symmetric and semipositive definite, there exists an $n \times n$ matrix $C$ such that

$$
A=C^{T} C \text {. }
$$

Then, by using Cauchy-Bunyakovsky inequality, we have

$$
|(C \zeta, C \eta)|^{2} \leq|C \zeta|^{2} \cdot|C \eta|^{2}
$$

It follows that

$$
\left|\zeta^{T} A \eta\right|^{2} \leq\left|\zeta^{T} A \zeta\right| \cdot\left|\eta^{T} A \eta\right|
$$

This proof is completed.

Lemma 2. Assume that $P$ is an $n \times n$ symmetric real matrix and $\lambda^{m}$ and $\lambda^{M}$ are the smallest and the largest eigenvalues of $P$, respectively. Then, for an arbitrary column vector $\xi \in R^{n}$, the following inequality holds:

$$
\lambda^{m} \xi^{T} \xi \leq \xi^{T} P \xi \leq \lambda^{M} \xi^{T} \xi
$$

Proof. $P$ is a symmetric real matrix; then, there exists an orthogonal matrix $U \in R^{n \times n}$ such that

$$
P=U \Lambda U^{T},
$$

where $\Lambda=\operatorname{diag}\left(\lambda_{1}, \lambda_{2}, \ldots, \lambda_{n}\right)$ and $\lambda_{i}$ is the $i$ th eigenvalue of $P, i=1,2, \ldots, n$.

For an arbitrary column vector $\xi \in R^{n}$, we have

$$
\begin{aligned}
\xi^{T} P \xi & =\left(U^{T} \xi\right)^{T} \Lambda\left(U^{T} \xi\right) \\
& =\sum_{i=1}^{n} \lambda_{i}\left|\left(U^{T} \xi\right)_{i}\right|^{2} \\
& \leq \lambda^{M} \sum_{i=1}^{n}\left|\left(U^{T} \xi\right)_{i}\right|^{2} \\
& =\lambda^{M} \sum_{i=1}^{n}\left|(\xi)_{i}\right|^{2} \\
& =\lambda^{M} \xi^{T} \xi .
\end{aligned}
$$

Similarly, we can prove

$$
\xi^{T} P \xi \geq \lambda^{m} \xi^{T} \xi
$$

The proof is completed.

\section{Impulsive Control of Unified Chaotic System}

Consider the unified chaotic system $[3,4,8,10]$ described by the following form:

$$
\begin{aligned}
& \dot{y}_{1}=(25 \alpha+10)\left(y_{2}-y_{1}\right), \\
& \dot{y}_{2}=(28-35 \alpha) y_{1}+(29 \alpha-1) y_{2}-y_{1} y_{3}, \\
& \dot{y}_{3}=y_{1} y_{2}-\frac{8+\alpha}{3} y_{3},
\end{aligned}
$$

where $y_{1}, y_{2}, y_{3}$ are state variables and $\alpha \in[0,1]$. System (9) has some special features and advantages because it unifies Lorenz system (when $\alpha \in[0,0.8)$ ), Lü system ( $\alpha=0.8)$, and Chen system (when $\alpha \in(0.8,1]$ ). Moreover, system (9) is always chaotic in the whole interval $\alpha \in[0,1]$.

It is easy to see that system (9) has three equilibrium points:

$$
\begin{gathered}
E_{1}=(0,0,0)^{T}, \quad E_{2}=(\beta, \beta, \eta)^{T}, \\
E_{3}=(-\beta,-\beta, \eta)^{T},
\end{gathered}
$$

where $\beta=\sqrt{(8+\alpha)(9-2 \alpha)}$ and $\eta=27-6 \alpha$.

For convenience, denote the above equilibrium points as $\left(y_{1}^{0}, y_{2}^{0}, y_{3}^{0}\right)^{T}$ and introduce the following transformation:

$$
x_{i}=y_{i}-y_{i}^{0}, \quad i=1,2,3 .
$$

Then, from (9), the following model can be obtained:

$$
\begin{gathered}
\dot{x}_{1}=(25 \alpha+10)\left(x_{2}-x_{1}\right), \\
\dot{x}_{2}=\left(28-35 \alpha-y_{3}^{0}\right) x_{1}+(29 \alpha-1) x_{2} \\
-y_{1}^{0} x_{3}-x_{1} x_{3}, \\
\dot{x}_{3}=y_{2}^{0} x_{1}+y_{1}^{0} x_{2}-\frac{8+\alpha}{3} x_{3}+x_{1} x_{2} .
\end{gathered}
$$

We decompose the linear and nonlinear parts of system (12) and rewrite it as the following form:

$$
\dot{x}=A x+\Phi(x),
$$

where $x=\left(x_{1}, x_{2}, x_{3}\right)^{T}$ and

$$
\begin{gathered}
A=\left(\begin{array}{ccc}
-(25 \alpha+10) & 25 \alpha+10 & 0 \\
28-35 \alpha-y_{3}^{0} & 29 \alpha-1 & -y_{1}^{0} \\
y_{2}^{0} & y_{1}^{0} & -\frac{\alpha+8}{3}
\end{array}\right), \\
\Phi(x)=\left(\begin{array}{c}
0 \\
-x_{1} x_{3} \\
x_{1} x_{2}
\end{array}\right) .
\end{gathered}
$$

In order to stabilize the equilibrium point, we now introduce a control model of system (13) described by

$$
\dot{x}(t)=A x(t)+\Phi(x(t))+\sum_{k=1}^{\infty} \delta\left(t-\tau_{k}\right) B_{k} x(t),
$$


where $\delta(\cdot)$ is the Dirac delta function, the time sequence $\left\{\tau_{k}\right\}$ satisfies $0=\tau_{0}<\tau_{1}<\tau_{2}<\cdots<\tau_{k}<\tau_{k+1} \cdots, \lim _{k \rightarrow \infty} \tau_{k}=$ $\infty$, and $B_{k}$ denotes a $3 \times 3$ constant matrix for each $k \in Z^{+}=$ $\{1,2, \ldots, n, n+1, \ldots\}$.

Integrating from $\tau_{k}-h$ to $\tau_{k}+h$ both sides of system (15), we have

$$
\begin{aligned}
& x\left(\tau_{k}+h\right)-x\left(\tau_{k}-h\right) \\
& \quad=\int_{\tau_{k}-h}^{\tau_{k}+h}\left[A x(t)+\Phi(x(t))+\sum_{k=1}^{\infty} \delta\left(t-\tau_{k}\right) B_{k} x(t)\right] d t,
\end{aligned}
$$

where $h>0$ is sufficiently small. As $h \rightarrow 0^{+}$, by applying the properties of the Dirac delta function, we have

$$
x\left(\tau_{k}^{+}\right)-x\left(\tau_{k}^{-}\right)=B_{k} x\left(\tau_{k}\right) .
$$

Throughout this paper, we always assume that $x(t)=$ $\left(x_{1}(t), x_{2}(t), x_{3}(t)\right)^{T}$ is left continuous at $\tau_{k}\left(k \in Z^{+}\right)$; that is, $x_{i}(t)=\lim _{t \rightarrow \tau_{k}^{-}} x_{i}(t)$. In this case, the above equality is equivalent to the following form:

$$
x\left(\tau_{k}^{+}\right)-x\left(\tau_{k}\right)=B_{k} x\left(\tau_{k}\right) .
$$

Thus, from the definition of the Dirac delta function, control system (15) can be rewritten as

$$
\begin{gathered}
\dot{x}(t)=A x(t)+\Phi(x(t)), \quad t \neq \tau_{k}, \\
x\left(t^{+}\right)-x(t)=B_{k} x(t), \quad t=\tau_{k} .
\end{gathered}
$$

It is easy to see that the origin is an equilibrium point of system (19). We are interested in stabilizing the origin of system (19), which is equivalent to the asymptotical stability of the equilibrium point $\left(y_{1}^{0}, y_{2}^{0}, y_{3}^{0}\right)^{T}$ of system (9).

For convenience, denote $\delta_{k}=\tau_{k+1}-\tau_{k}, \Delta_{k}=\tau_{2 k+1}-$ $\tau_{2 k-1}\left(k \in Z^{+}\right)$and assume that

$$
\sup _{k \in Z^{+}}\left\{\delta_{k}\right\}=\sigma<\infty
$$

Since system (9) is chaotic, there exist two positive numbers $S_{1}$ and $S_{2}$ such that $\left|y_{1}(t)\right| \leq S_{1},\left|y_{2}(t)\right| \leq S_{2}$, and $\left|y_{3}(t)\right| \leq S_{2}$ for $t \in R^{+}$(see [4]). Additionally, we have $\left|x_{1}(t)\right| \leq S_{1}+\left|y_{1}^{0}\right|=$ $M_{1},\left|x_{2}(t)\right| \leq S_{2}+\left|y_{2}^{0}\right| \leq S_{2}+\left|y_{3}^{0}\right|=M_{2}$, and $\left|x_{3}(t)\right| \leq$ $S_{2}+\left|y_{3}^{0}\right|=M_{2}$ for $t \in R^{+}$.

The following theorem is provided to ensure the globally asymptotical stability of the origin for the impulsive control system (19).

Theorem 3. Let $P$ be a $3 \times 3$ symmetric and positive definite matrix such that $(P-I)$ is semipositive definite, where I denotes the identity matrix. Denote that $\lambda_{1}$ and $\lambda_{2}$ are the smallest and the largest eigenvalues of $P$ and $(P-I)$, respectively. Let $q$ be the largest eigenvalue of $\left(P A+A^{T} P\right) \cdot d_{k}$ is the largest eigenvalue of the matrix $\left(I+B_{k}\right)^{T} P\left(I+B_{k}\right)$ and $\alpha=\inf _{k \in Z^{+}}\left\{d_{k}\right\}>0$, $\zeta=\sup _{k \in Z^{+}}\left\{d_{k}\right\}<\infty$. Denote that

$$
\gamma= \begin{cases}0, & P=I, \\ \frac{2 M_{1} \lambda_{2}}{\lambda_{1}}, & P \neq I\end{cases}
$$

The origin of the controlled unified chaotic system (19) is globally asymptotically stable if there exist two constants $\mu>1$ and $b \geq 0$ such that one of the following conditions is satisfied.

(1) For all $k \in Z^{+}$,

$$
\left(\frac{q}{\lambda_{1}}+\gamma-b\right) \delta_{k}+\ln \left(\frac{\mu d_{k}}{\lambda_{1}}\right) \leq 0 .
$$

(2) For all $k \in Z^{+}$,

$$
\left(\frac{q}{\lambda_{1}}+\gamma-b\right) \Delta_{k}+\ln \left(\frac{\mu d_{2 k} d_{2 k-1}}{\lambda_{1}^{2}}\right) \leq 0 .
$$

Proof. Firstly, construct an auxiliary function described by the following form:

$$
\begin{gathered}
V(t, x)=e^{b\left(\tau_{k-1}-t\right)} x^{T}(t) P x(t), \\
t \in\left(\tau_{k-1}, \tau_{k}\right], \quad k=1,2, \ldots
\end{gathered}
$$

Denote that $Q=A^{T} P+P A$. For $t \in\left(\tau_{k-1}, \tau_{k}\right]$, if $P \neq I$, in view of Lemmas 1 and 2, the derivative of $V(t)$ along the solution of (19) is

$$
\begin{aligned}
D^{+} V(t) & =e^{b\left(\tau_{k-1}-t\right)}\left[x^{T} Q x+x^{T} P \Phi(x)+\Phi^{T}(x) P x\right]-b V \\
& =e^{b\left(\tau_{k-1}-t\right)}\left[x^{T} Q x+2 x^{T}(P-I) \Phi(x)\right]-b V \\
& \leq e^{b\left(\tau_{k-1}-t\right)}\left(x^{T} Q x+2 \sqrt{x^{T}(P-I) x}\right. \\
& \left.\times \sqrt{\Phi^{T}(x)(P-I) \Phi(x)}\right)-b V \\
\leq & e^{b\left(\tau_{k-1}-t\right)}\left(x^{T} Q x+2 \lambda_{2} \sqrt{x^{T} x} \sqrt{\Phi^{T}(x) \Phi(x)}\right)-b V \\
\leq & e^{b\left(\tau_{k-1}-t\right)}\left(x^{T} Q x+2 \lambda_{2}\left|x_{1}\right| x^{T} x\right)-b V \\
\leq & e^{b\left(\tau_{k-1}-t\right)}\left(\frac{q}{\lambda_{1}}+\frac{2 \lambda_{2} M_{1}}{\lambda_{1}}\right) x^{T} P x-b V \\
& =\left(\frac{q}{\lambda_{1}}+\frac{2 \lambda_{2} M_{1}}{\lambda_{1}}-b\right) V(t) .
\end{aligned}
$$

On the other hand, if $P=I$,

$$
\begin{aligned}
D^{+} V(t) & =e^{b\left(\tau_{k-1}-t\right)} x^{T} Q x-b V \\
& \leq \frac{q}{\lambda_{1}} e^{b\left(\tau_{k-1}-t\right)} x^{T} P x-b V \\
& =\left(\frac{q}{\lambda_{1}}-b\right) V(t) .
\end{aligned}
$$

From what has been discussed above, we have

$$
D^{+} V(t) \leq\left(\frac{q}{\lambda_{1}}+\gamma-b\right) V(t) .
$$


Besides, for $t=\tau_{k}$,

$$
\begin{aligned}
V\left(\tau_{k}^{+}\right) & =e^{b\left(\tau_{k-1}-\tau_{k}\right)} x^{T}\left(\tau_{k}^{+}\right) \operatorname{Px}\left(\tau_{k}^{+}\right) \\
& =e^{b\left(\tau_{k-1}-\tau_{k}\right)} x^{T}\left(\tau_{k}\right)\left(I+B_{k}\right)^{T} P\left(I+B_{k}\right) x\left(\tau_{k}\right) \\
& \leq \frac{d_{k}}{\lambda_{1}} e^{b\left(\tau_{k-1}-\tau_{k}\right)} x^{T}\left(\tau_{k}\right) P x\left(\tau_{k}\right) \\
& =\frac{d_{k}}{\lambda_{1}} V\left(\tau_{k}\right) .
\end{aligned}
$$

From (27), for $t \in\left[0, \tau_{1}\right]$,

$$
V(t) \leq V(0) \exp \left[\left(\frac{q}{\lambda_{1}}+\gamma-b\right) t\right],
$$

which leads to

$$
V\left(\tau_{1}\right) \leq V(0) \exp \left[\left(\frac{q}{\lambda_{1}}+\gamma-b\right) \tau_{1}\right] .
$$

Further, by using (28),

$$
V\left(\tau_{1}^{+}\right) \leq V(0) \frac{d_{1}}{\lambda_{1}} \exp \left[\left(\frac{q}{\lambda_{1}}+\gamma-b\right) \tau_{1}\right] .
$$

Similarly, for $t \in\left(\tau_{1}, \tau_{2}\right]$, we obtain

$$
\begin{aligned}
V(t) & \leq V\left(\tau_{1}^{+}\right) \exp \left[\left(\frac{q}{\lambda_{1}}+\gamma-b\right)\left(t-\tau_{1}\right)\right] \\
& \leq V(0) \frac{d_{1}}{\lambda_{1}} \exp \left[\left(\frac{q}{\lambda_{1}}+\gamma-b\right) t\right], \\
V\left(\tau_{2}^{+}\right) & \leq V(0) \frac{d_{1}}{\lambda_{1}} \frac{d_{2}}{\lambda_{1}} \exp \left[\left(\frac{q}{\lambda_{1}}+\gamma-b\right) \tau_{2}\right] .
\end{aligned}
$$

Hence, generally, for $t \in\left(\tau_{k}, \tau_{k+1}\right]$,

$$
V(t) \leq V(0)\left(\prod_{i=1}^{k} \frac{d_{i}}{\lambda_{1}}\right) \exp \left[\left(\frac{q}{\lambda_{1}}+\gamma-b\right) t\right]
$$

If $q / \lambda_{1}+\gamma-b \geq 0$, then

$$
\begin{aligned}
V(t) \leq & V(0)\left(\prod_{i=1}^{k} \frac{d_{i}}{\lambda_{1}}\right) \exp \left[\left(\frac{q}{\lambda_{1}}+\gamma-b\right) \tau_{k+1}\right] \\
= & V(0) \exp \left[\left(\frac{q}{\lambda_{1}}+\gamma-b\right) \tau_{1}\right] \\
& \times \prod_{i=1}^{k} \frac{d_{i}}{\lambda_{1}} \exp \left(\frac{q}{\lambda_{1}}+\gamma-b\right)\left(\tau_{i+1}-\tau_{i}\right) .
\end{aligned}
$$

If $q / \lambda_{1}+\gamma-b<0$, then

$$
\begin{aligned}
V(t) \leq & V(0)\left(\prod_{i=1}^{k} \frac{d_{i}}{\lambda_{1}}\right) \exp \left[\left(\frac{q}{\lambda_{1}}+\gamma-b\right) \tau_{k+1}\right] \\
& \times \exp \left[\left(\frac{q}{\lambda_{1}}+\gamma-b\right)\left(t-\tau_{k+1}\right)\right] \\
\leq & V(0) \beta\left(\prod_{i=1}^{k} \frac{d_{i}}{\lambda_{1}}\right) \exp \left[\left(\frac{q}{\lambda_{1}}+\gamma-b\right) \tau_{k+1}\right] \\
= & V(0) \beta \exp \left[\left(\frac{q}{\lambda_{1}}+\gamma-b\right) \tau_{1}\right] \\
& \times \prod_{i=1}^{k} \frac{d_{i}}{\lambda_{1}} \exp \left(\frac{q}{\lambda_{1}}+\gamma-b\right)\left(\tau_{i+1}-\tau_{i}\right),
\end{aligned}
$$

where

$$
\beta=\exp \left[\left|\frac{q}{\lambda_{1}}+\gamma-b\right| \sigma\right]>1 .
$$

Hence, for $t \in\left(\tau_{k}, \tau_{k+1}\right]$, we always have

$$
\begin{aligned}
V(t) \leq & V(0) \beta \exp \left[\left(\frac{q}{\lambda_{1}}+\gamma-b\right) \tau_{1}\right] \\
& \times \prod_{i=1}^{k} \frac{d_{i}}{\lambda_{1}} \exp \left(\frac{q}{\lambda_{1}}+\gamma-b\right)\left(\tau_{i+1}-\tau_{i}\right) .
\end{aligned}
$$

In the following, we consider the following cases.

Case 1. When condition (9) is satisfied, from (22),

$$
\frac{d_{i}}{\lambda_{1}} \exp \left(\frac{q}{\lambda_{1}}+\gamma-b\right)\left(\tau_{i+1}-\tau_{i}\right) \leq \frac{1}{\mu}, \quad i \in Z^{+},
$$

and together with (37), we have

$$
V(t) \leq \frac{1}{\mu^{k}} V(0) \beta \exp \left[\left(\frac{q}{\lambda_{1}}+\gamma-b\right) \tau_{1}\right] .
$$

Note that $k \rightarrow \infty$ as $t \rightarrow \infty$; then, from $\mu>1$, we obtain $\lim _{t \rightarrow \infty} V(t)=0$. Therefore, we finally have $\lim _{t \rightarrow \infty} x(t)=0$.

Case 2. If condition (12) holds, from (23), we have

$$
\frac{d_{2 i} d_{2 i-1}}{\lambda_{1}^{2}} \exp \left(\frac{q}{\lambda_{1}}+\gamma-b\right)\left(\tau_{2 i+1}-\tau_{2 i-1}\right) \leq \frac{1}{\mu}, \quad i \in Z^{+} .
$$

For $t \in\left(\tau_{2 k-1}, \tau_{2 k}\right]$, it follows from (37) and (40) that

$$
\begin{aligned}
V(t) \leq & \frac{1}{\mu^{k-1}} \beta V(0) \exp \left[\left(\frac{q}{\lambda_{1}}+\gamma-b\right) \tau_{1}\right] \\
& \times \frac{d_{2 k-1}}{\lambda_{1}} \exp \left(\frac{q}{\lambda_{1}}+\gamma-b\right)\left(\tau_{2 k}-\tau_{2 k-1}\right) \\
\leq & \frac{1}{\mu^{k-1}} V(0) \beta \exp \left[\left|\frac{q}{\lambda_{1}}+\gamma-b\right|\left(\tau_{1}+\sigma\right)\right] \frac{\zeta}{\lambda_{1}} .
\end{aligned}
$$


Similarly, if $t \in\left(\tau_{2 k}, \tau_{2 k+1}\right]$, from (37) and (40), we can derive that

$$
V(t) \leq \frac{1}{\mu^{k}} V(0) \beta \exp \left[\left(\frac{q}{\lambda_{1}}+\gamma-b\right) \tau_{1}\right] .
$$

From (41) and (42), we finally have $\lim _{t \rightarrow \infty} x(t)=0$.

From the above analysis, the trivial solution $x(t)=0$ of system (19) is globally asymptotically stable. The proof of Theorem 3 is completed.

Remark 4. From Theorem 3, it is clear that $\gamma=0$ if $P=I$. On the other hand, for $P \neq I, \gamma \rightarrow 0$ as $P$ approaches to $I$, since $\lambda_{2} \rightarrow 0$ in this case.

Remark 5. If $\mu=1$ in Theorem 3, from the proof of Theorem 3, we have

$$
V(t) \leq V(0) \exp \left[\left(\frac{q}{\lambda_{1}}+\gamma-b\right) \tau_{1}\right] \beta Q,
$$

where $Q=\max \left\{1,\left(\zeta / \lambda_{1}\right) \exp \left[\left|q / \lambda_{1}+\gamma-b\right| \sigma\right]\right\}$. In this case, it is clear that the origin of impulsive control system (19) is stable.

Taking $P=I$ in Theorem 3 , it is easy to see that $\lambda_{1}=1$, $\lambda_{2}=0$, and $\gamma=0$ in this case. By virtue of Theorem 3, the following statements can be derived.

Corollary 6. The origin of the controlled unified chaotic system (19) is globally asymptotically stable if there exist two constants $\mu>1$ and $b \geq 0$ such that one of the following conditions holds.

(a) For all $k \in Z^{+}$,

$$
(q-b) \delta_{k}+\ln \left(\mu d_{k}\right) \leq 0 .
$$

(b) For all $k \in Z^{+}$,

$$
(q-b) \Delta_{k}+\ln \left(\mu d_{2 k} d_{2 k-1}\right) \leq 0, \quad k \in Z^{+} .
$$

Remark 7. In Corollary 6, if $b=0$, inequality (44) is reduced to

$$
q \delta_{k}+\ln \left(\mu d_{k}\right) \leq 0
$$

which is equivalent to the condition of Theorem 1 in [10]. Further, in this case, inequality (45) is degenerated to

$$
q \Delta_{k}+\ln \left(\mu d_{2 k} d_{2 k-1}\right) \leq 0
$$

which is the same as inequality (7) in [8]. On the other hand, for $b>0$, evidently, conditions (44) and (45) are less conservative than inequalities (46) and (47). Hence, compared with $[8,10]$, our results are less stringent and more general.

In practice, the equal impulsive interval and the constant gain matrix are often selected for convenience. In Corollary 6 , we choose $\delta_{i}=\delta$ and $B_{i}=B$ for $i \in Z^{+}$and denote that $q$ and $d$ are the largest eigenvalues of $\left(A^{T}+A\right)$ and $(I+B)^{T}(I+B)$, respectively. Then, the following corollary can be obtained.
Corollary 8. The origin of system (19) is globally asymptotically stable if there exist $\mu>1$ and $b \geq 0$ such that

$$
(q-b) \delta+\ln (\mu d) \leq 0 .
$$

Remark 9. From Corollary 8, an upper bound of equal impulsive interval $\delta$ can be derived. In fact,

$$
\delta \leq \frac{1}{q-b} \ln \frac{1}{\mu d} .
$$

In [10], the upper bound of impulsive interval was also given, which can be represented by

$$
\delta \leq \frac{1}{q} \ln \frac{1}{\mu d} .
$$

It is evident that the equal impulsive interval obtained in this paper is larger, which shows that our designed controller is more efficient and more economical in practice.

\section{Impulsive Synchronization of Unified Chaotic System}

In this section, we discuss the impulsive synchronization of unified chaotic system. It is easy to see that system (9) can be rewritten as

$$
\dot{y}=A y+\Phi(y)
$$

where $y=\left(y_{1}, y_{2}, y_{3}\right)^{T}$ and

$$
\begin{gathered}
A=\left(\begin{array}{ccc}
-(25 \alpha+10) & 25 \alpha+10 & 0 \\
28-35 \alpha & 29 \alpha-1 & 0 \\
0 & 0 & -\frac{\alpha+8}{3}
\end{array}\right), \\
\Phi(y)=\left(\begin{array}{c}
0 \\
-y_{1} y_{3} \\
y_{1} y_{2}
\end{array}\right) .
\end{gathered}
$$

In impulsive synchronization configuration, the driving system is given by (51), while the driven system is represented by the following form:

$$
\dot{\tilde{y}}=A \tilde{y}+\Phi(\tilde{y})+\sum_{k=1}^{\infty} \delta\left(t-\tau_{k}\right) B_{k}(\tilde{y}(t)-y(t)),
$$

where $\tilde{y}=\left(\tilde{y}_{1}, \tilde{y}_{2}, \tilde{y}_{3}\right)^{T}$ is the state variable of the driven system, $A$ and $\Phi$ are defined in (52), and $B_{k}, \delta(\cdot)$, and $\left\{\tau_{k}\right\}$ are defined in (15).

Similar to the technique of system (15), the driven system (53) can be rewritten as the following impulsive equation:

$$
\begin{gathered}
\dot{\tilde{y}}=A \tilde{y}+\Phi(\tilde{y}), \quad t \neq \tau_{k}, \\
\Delta \tilde{y}=B_{k}(\tilde{y}(t)-y(t)), \quad t=\tau_{k} .
\end{gathered}
$$

Let $e=\left(e_{1}, e_{2}, e_{3}\right)^{T}=\left(\tilde{y}_{1}-y_{1}, \tilde{y}_{2}-y_{2}, \tilde{y}_{3}-y_{3}\right)^{T}$ be the synchronization error and

$$
\Psi(y, \tilde{y})=\Phi(\tilde{y})-\Phi(y)=\left(\begin{array}{c}
0 \\
y_{1} y_{3}-\tilde{y}_{1} \tilde{y}_{3} \\
\tilde{y}_{1} \widetilde{y}_{2}-y_{1} y_{2}
\end{array}\right)
$$


From (51) and (54), the error system can be expressed by

$$
\begin{gathered}
\dot{e}=A e+\Psi(y, \tilde{y}), \quad t \neq \tau_{k}, \\
\Delta e=B_{k} e, \quad t=\tau_{k} .
\end{gathered}
$$

In this section, we are interested in synchronizing the driving system (51) and the driven system (54), which is equivalent to the asymptotical stability of the origin for error system (56). Similar to Theorem 3, the following results are stated, which ensure the globally asymptotical synchronization of the driving system (51) and the driven system (54).

Theorem 10. Let $q$ be the largest eigenvalue of $\left(A+A^{T}\right) \cdot d_{k}$ is the largest eigenvalue of the matrix $\left(I+B_{k}\right)^{T}\left(I+B_{k}\right)$. The driving system (51) and the driven system (54) are globally asymptotically synchronized if there exist constants $\mu>1$ and $b \geq 0$ such that one of the following conditions is satisfied.

(a) For all $k \in Z^{+}$,

$$
\left(q+2 S_{2}-b\right) \delta_{k}+\ln \left(\mu d_{k}\right) \leq 0 .
$$

(b) For all $k \in Z^{+}$,

$$
\left(q+2 S_{2}-d\right) \Delta_{k}+\ln \left(\mu d_{2 k} d_{2 k-1}\right) \leq 0 .
$$

Proof. Observe that error system (56) is almost the same as system (19) except for $\Psi(y, \tilde{y})$. Construct an auxiliary function described by the following form:

$$
\begin{aligned}
& V(t, x)=e^{b\left(\tau_{k-1}-t\right)} e^{T}(t) e(t), \\
& t \in\left(\tau_{k-1}, \tau_{k}\right], \quad k=1,2, \ldots
\end{aligned}
$$

For $t \in\left(\tau_{k-1}, \tau_{k}\right]$, calculating the time derivative of $V(t)$ along the solution of (56), we have

$$
\begin{aligned}
& D^{+} V(t) \\
& =e^{b\left(\tau_{k-1}-t\right)}\left[e^{T}\left(A^{T}+A\right) e+e^{T} \Psi(y, \tilde{y})+\Psi^{T}(y, \tilde{y}) e\right]-b V \\
& =e^{b\left(\tau_{k-1}-t\right)}\left[e^{T}\left(A^{T}+A\right) e+2\left(y_{2} e_{1} e_{3}-y_{3} e_{1} e_{2}\right)\right]-b V \\
& \leq e^{b\left(\tau_{k-1}-t\right)}\left[e^{T}\left(A^{T}+A\right) e+2 S_{2} e^{T} e\right]-b V \\
& \leq e^{b\left(\tau_{k-1}-t\right)}\left(q+2 S_{2}\right) e^{T} e-b V \\
& \leq\left(q+2 S_{2}-b\right) V .
\end{aligned}
$$

In addition, for $k \in Z^{+}$,

$$
\begin{aligned}
V\left(\tau_{k}^{+}\right) & =e^{b\left(\tau_{k-1}-\tau_{k}\right)} e^{T}\left(\tau_{k}\right)\left(I+B_{k}\right)^{T}\left(I+B_{k}\right) e\left(\tau_{k}\right) \\
& \leq d_{k} V\left(\tau_{k}\right) .
\end{aligned}
$$

The rest of this proof is the same as that of Theorem 3, and here we omit it. The proof of Theorem 10 is completed.

Similar to Corollary 6 , we have the following results.

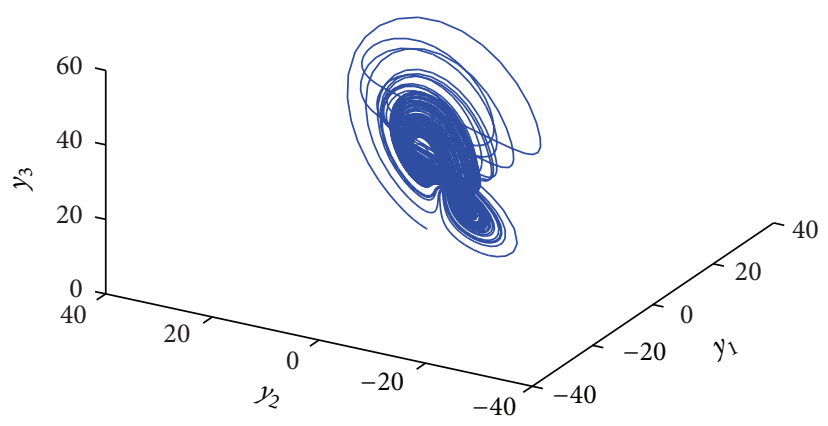

FIgURE 1: The chaotic behavior of system (9) with $\alpha=1$.

Corollary 11. The driving system (51) and the driven system (54) are globally asymptotically synchronized, if there exist $\mu>$ 1 and $b \geq 0$ such that one of the following conditions is satisfied.

(a) For all $k \in Z^{+}$,

$$
\left(q+2 S_{2}-b\right) \delta_{k}+\ln \left(\mu d_{k}\right) \leq 0 .
$$

(b) For all $k \in Z^{+}$,

$$
\left(q+2 S_{2}-b\right) \Delta_{k}+\ln \left(\mu d_{2 k} d_{2 k-1}\right) \leq 0, \quad k \in Z^{+} .
$$

Remark 12. The impulsive synchronization of unified chaotic system was investigated in $[8,10]$. It is noted that inequality (62) is reduced to the condition of Theorem 2 in [10] and condition (b) in Corollary 11 will be degenerated to the assumption of Theorem 2 in [8] when $b=0$. Additionally, if $b>0$, it is evident that conditions (62) and (63) are less stringent than the assumptions introduced in $[8,10]$.

Similar to Corollary 8 , the following results can be derived when we select $\delta_{i}=\delta$ and $B_{i}=B$ for each $k \in Z^{+}$.

Corollary 13. The driving system (51) and the driven system (54) are globally asymptotically synchronized, if there exist $\mu>$ 1 and $b \geq 0$ such that

$$
\left(q+2 S_{2}-b\right) \delta+\ln (\mu d) \leq 0,
$$

where $d$ is the largest eigenvalue of $(I+B)^{T}(I+B)$.

\section{Numerical Simulations}

In this section, based on the results obtained in the previous sections, some numerical simulations are represented to show the effectiveness of our results.

First, we consider numerical simulations of impulsive control for system (9) with $\alpha=1$. The chaotic behavior of system (9) with $\alpha=1$ is shown in Figure 1.

For convenience, select $\left(y_{1}^{0}, y_{2}^{0}, y_{3}^{0}\right)=(0,0,0), P=I$ and take gain matrices $B_{i}=\operatorname{diag}(-0.86,-0.84,-0.9)\left(i \in Z^{+}\right)$in Theorem 3. By simple computation, we obtain

$$
A+A^{T}=\left(\begin{array}{ccc}
-70 & 28 & 0 \\
28 & 56 & 0 \\
0 & 0 & -6
\end{array}\right),
$$



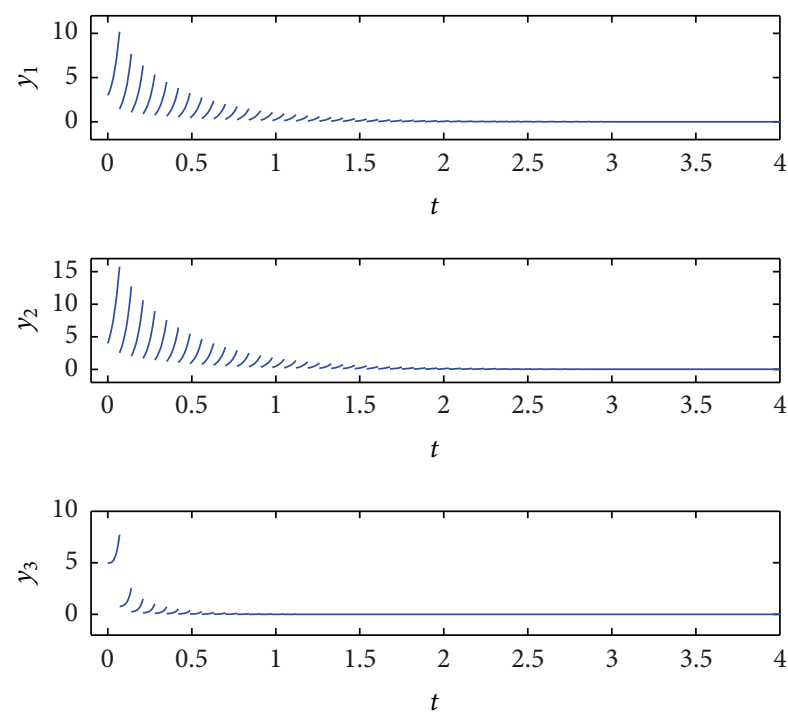

FIGURE 2: Impulsive stabilization of system (9) with $\alpha=1$ and $\delta=$ 0.07 .

and its eigenvalues are $-75.942,-6$, and 61.942; then, $q=$ 61.942 .

Choosing impulsive control interval $\delta=0.07, \mu=1.1$. By computation, $d=0.0256$ and

$$
\begin{aligned}
q \delta+\ln (\mu d) & =61.942 \times 0.07+\ln (1.1 \times 0.0256) \\
& =0.7661 .
\end{aligned}
$$

Taking $b=12$ in Theorem 3, then,

$$
b \delta=12 \delta=0.84 \text {. }
$$

Hence,

$$
(q-b) \delta+\ln (\mu d)=-0.0739<0 .
$$

Therefore, all conditions of Corollary 8 are satisfied, which implies that the origin of unified chaotic system (9) is globally asymptotically stable under impulsive control. The numerical simulation results with constant impulsive interval $\delta=0.07$ are shown in Figure 2.

In the second simulation, we study impulsive synchronization of the driving system (51) and the driven system (54) with $\alpha=0$. The initial conditions of the driving system and the driven system are $(-2,-8,-1)$ and $(5,-9,-6)$, respectively. Figure 3 shows that system (51) with $\alpha=0$ is chaotic, and from this we can obtain that $S_{2}=65$.

Choose $B_{i}=\operatorname{diag}(-0.88,-0.8,-0.8), \delta=0.02, \mu=1.5$, and $b=20$ in (43). By computation, $q=28.0512, d=0.04$ and

$$
\left(q+2 S_{2}\right) \delta+\ln (\mu d)=0.3476
$$

and $b \delta=0.4$, which leads to

$$
\left(q+2 S_{2}-b\right) \delta+\ln (\mu d)=-0.0524 .
$$

By the above analysis, all conditions of Corollary 13 hold; hence, system (51) and system (54) are globally asymptotically

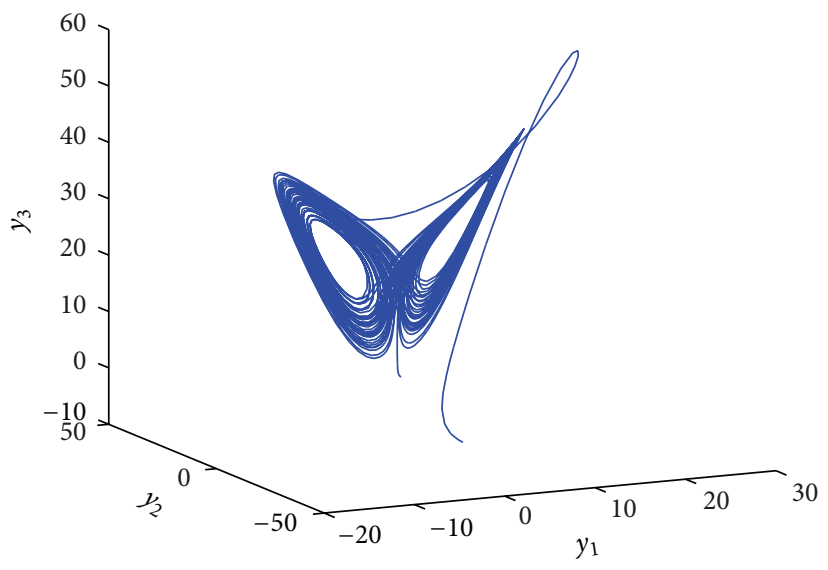

FIGURE 3: The chaotic behavior of system (51) with $\alpha=0$.
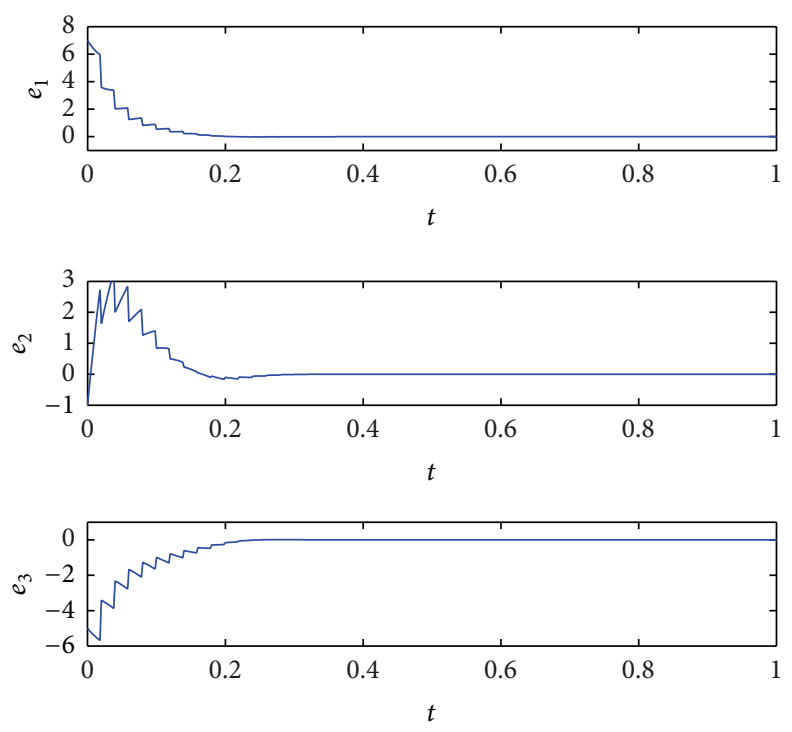

FIGURE 4: Synchronization error curves with $\alpha=0$ and $\delta=0.02$.

synchronized under impulsive control. The synchronization errors with impulsive distance $\delta=0.02$ are shown in Figure 4 .

Remark 14. Evidently, it follows from (66) and (69) that the conditions of Theorems 1 and 2 in [10] are not satisfied under the above parameters; that is to say, the stabilization and synchronization of unified chaotic system are not ensured by means of the criteria obtained in [10]. However, it follows from (68) and (70) that the stabilization and synchronization of unified chaotic system can be realized by virtue of our criteria and Figures 2 and 4 show the validity of our results. Therefore, our results are less conservative and more superior compared with [10].

\section{Conclusion}

In this paper, the issue on the stabilization and synchronization of unified chaotic system under impulsive control is investigated. Some novel and useful criteria are derived 
by using impulsive control theory. Finally, the effectiveness and feasibility of the developed methods are shown by some numerical simulations. Compared with some previous results given in $[8,10]$, our designed controller is more efficient and economical.

\section{Conflict of Interests}

The authors declare that there is no conflict of interests regarding the publication of this paper.

\section{Acknowledgments}

This work was supported by the Natural Science Foundation of Xinjiang (Grant no. 2013211B06), the Project funded by China Postdoctoral Science Foundation (Grant no. 2013M540782), the Natural Science Foundation of Xinjiang University (Grant no. BS120101), the Specialized Research Fund for the Doctoral Program of Higher Education (Grant no. 20136501120001), and the National Natural Science Foundation of China (Grant no. 61164004).

\section{References}

[1] W. Xie, C. Wen, and Z. Li, "Impulsive control for the stabilization and synchronization of Lorenz systems," Physics Letters A: General, Atomic and Solid State Physics, vol. 275, no. 1-2, pp. 6772, 2000.

[2] G. Chen and T. Ueta, "Yet another chaotic attractor," International Journal of Bifurcation and Chaos, vol. 9, no. 7, pp. 14651466, 1999.

[3] C. Tao, H. Xiong, and F. Hu, "Two novel synchronization criterions for a unified chaotic system," Chaos Solitons and Fractals, vol. 27, pp. 111-114, 2006.

[4] X. Wang and J. Song, "Synchronization of the unified chaotic system," Nonlinear Analysis: Theory, Methods and Applications, vol. 69, no. 10, pp. 3409-3416, 2008.

[5] J. Ma, B. Ren, and Y. Chen, "Impulsive control of chaotic attractors in nonlinear chaotic systems," Applied Mathematics and Mechanics (English Edition), vol. 25, no. 9, pp. 971-976, 2004.

[6] X. Guan, Z. Fan, H. Peng, and Y. Wang, "The adaptive control of Chen's chaotic system," Acta Physica Sinica, vol. 50, no. 11, pp. 2110-2111, 2001.

[7] X. Liu and S. Zhong, "T-S fuzzy model-based impulsive control of chaotic systems with exponential decay rate," Physics Letters A: General, Atomic and Solid State Physics, vol. 370, no. 3-4, pp. 260-264, 2007.

[8] D. Chen, J. Sun, and C. Huang, "Impulsive control and synchronization of general chaotic system," Chaos, Solitons and Fractals, vol. 28, no. 1, pp. 213-218, 2006.

[9] D. Chen, J. Sun, and Q. Wu, "Impulsive control and its application to Lü's chaotic system," Chaos, Solitons and Fractals, vol. 21, no. 5, pp. 1135-1142, 2004.

[10] S. Chen, Q. Yang, and C. Wang, "Impulsive control and synchronization of unified chaotic system," Chaos, Solitons and Fractals, vol. 20, no. 4, pp. 751-758, 2004.

[11] G. Jiang, Q. Lu, and L. Qian, "Chaos and its control in an impulsive differential system," Chaos, Solitons and Fractals, vol. 34, no. 4, pp. 1135-1147, 2007.
[12] C. Li, X. Liao, and R. Zhang, "Impulsive synchronization of nonlinear coupled chaotic systems," Physics Letters A: General, Atomic and Solid State Physics, vol. 328, no. 1, pp. 47-50, 2004.

[13] Z. G. Li, C. Y. Wen, and Y. C. Soh, "Analysis and design of impulsive control systems," IEEE Transactions on Automatic Control, vol. 46, no. 6, pp. 894-897, 2001.

[14] X. Liu, Y. Liu, and K. L. Teo, "Stability analysis of impulsive control systems," Mathematical and Computer Modelling, vol. 37, no. 12-13, pp. 1357-1370, 2003.

[15] J. Sun, "Impulsive control of a new chaotic system," Mathematics and Computers in Simulation, vol. 64, no. 6, pp. 669-677, 2004.

[16] J. Sun and Y. Zhang, "Impulsive control and synchronization of Chua's oscillators," Mathematics and Computers in Simulation, vol. 66, no. 6, pp. 499-508, 2004.

[17] J. Sun, Y. Zhang, and Q. Wu, "Less conservative conditions for asymptotic stability of impulsive control systems," IEEE Transactions on Automatic Control, vol. 48, no. 5, pp. 829-831, 2003.

[18] T. Yang, "Impulsive control," IEEE Transactions on Automatic Control, vol. 44, no. 5, pp. 1081-1083, 1999.

[19] X. Zhang, X. Liao, and C. Li, "Impulsive control, complete and lag synchronization of unified chaotic system with continuous periodic switch," Chaos, Solitons and Fractals, vol. 26, no. 3, pp. 845-854, 2005.

[20] Q. Zhang and J. Lu, "Impulsive control and synchronization of a critical chaotic system," Wuhan University Journal of Natural Sciences, vol. 12, no. 3, pp. 426-430, 2007.

[21] Y. Zhang and J. Sun, "Controlling chaotic Lu systems using impulsive control," Physics Letters A: General, Atomic and Solid State Physics, vol. 342, no. 3, pp. 256-262, 2005.

[22] J. Zhou, X. H. Cheng, and Y. C. Zhang, "Impulsive control and synchronization of chaotic systems consisting of Van der Pol oscillators coupled to linear oscillators," Chaos, Solitons and Fractals, vol. 33, pp. 607-616, 2007.

[23] V. Laskshmikantham, D. Bainov, and P. S. Simeonov, Theory of Impulsive Differential Equations, World Scientific, Singapore, 1989. 


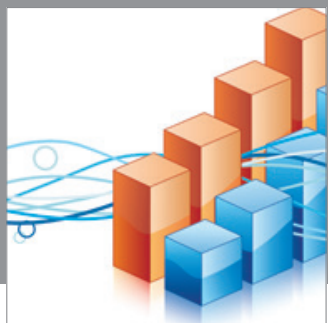

Advances in

Operations Research

mansans

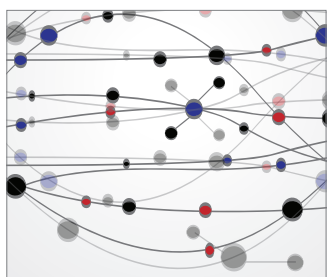

The Scientific World Journal
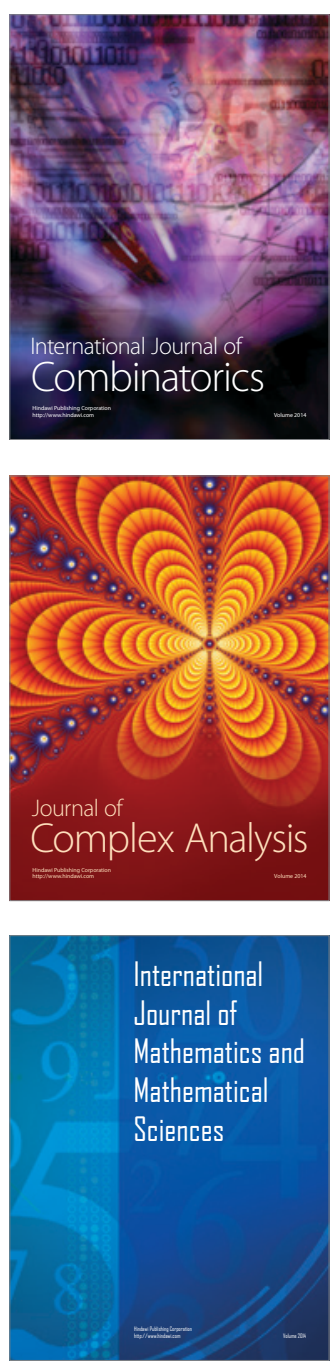
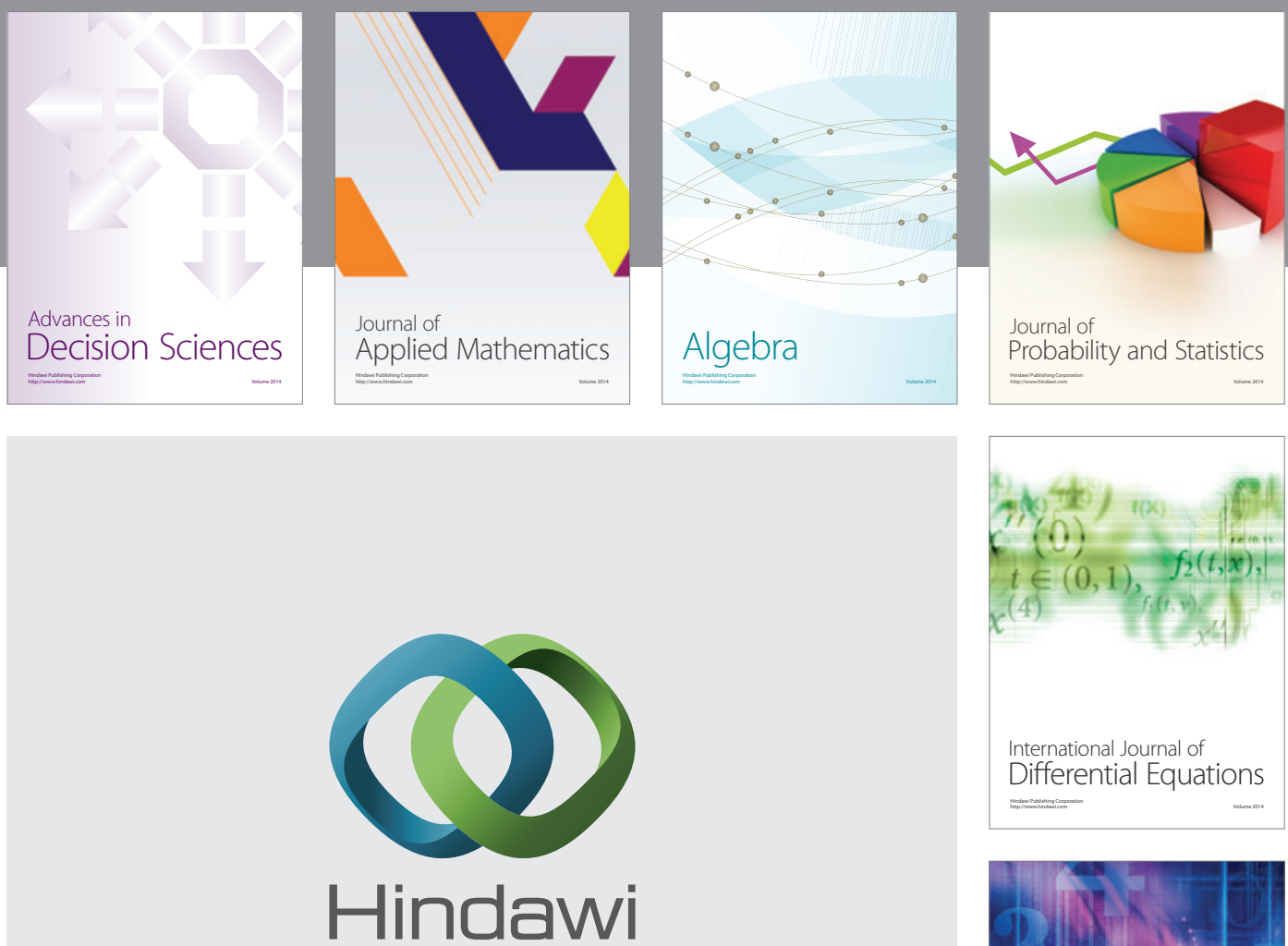

Submit your manuscripts at http://www.hindawi.com
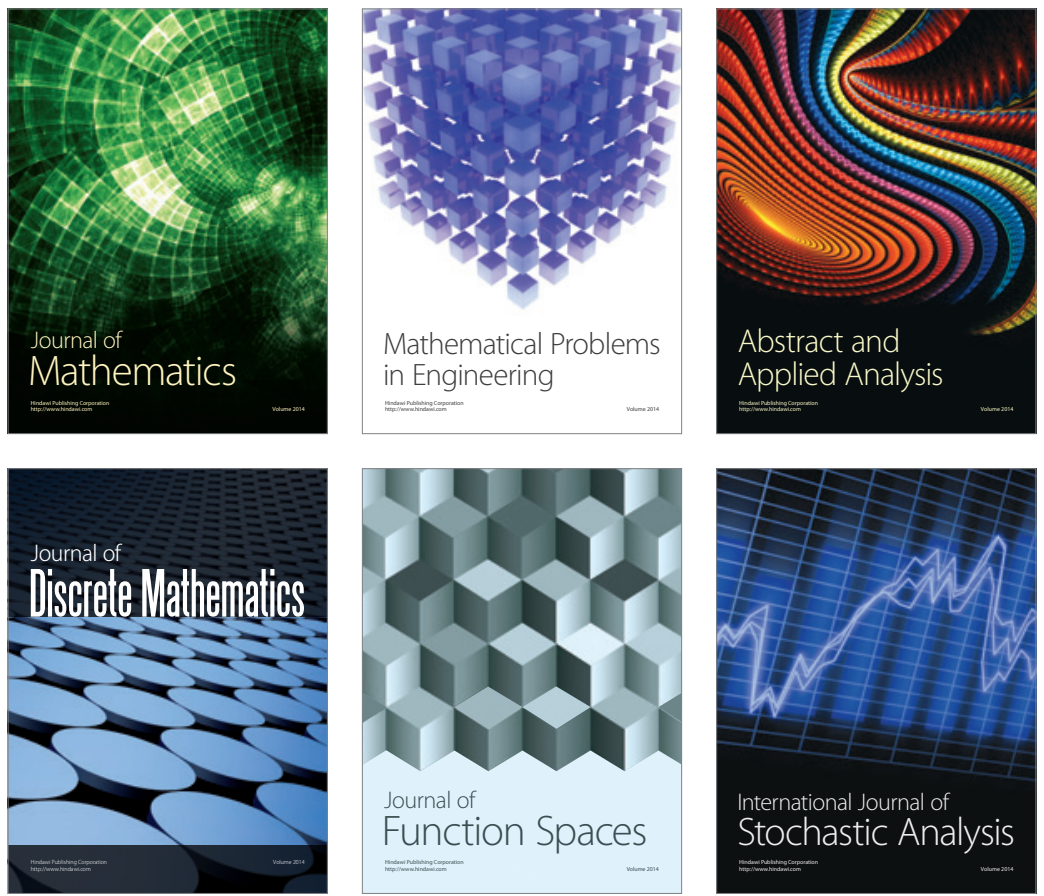

Journal of

Function Spaces

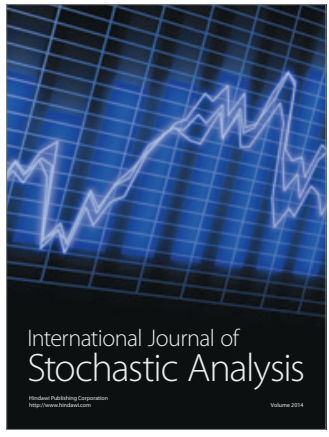

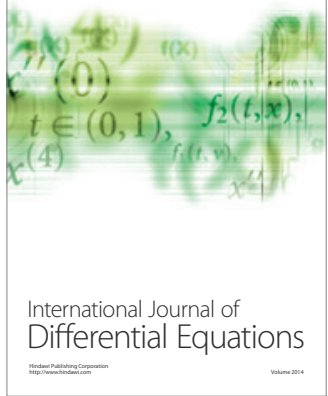
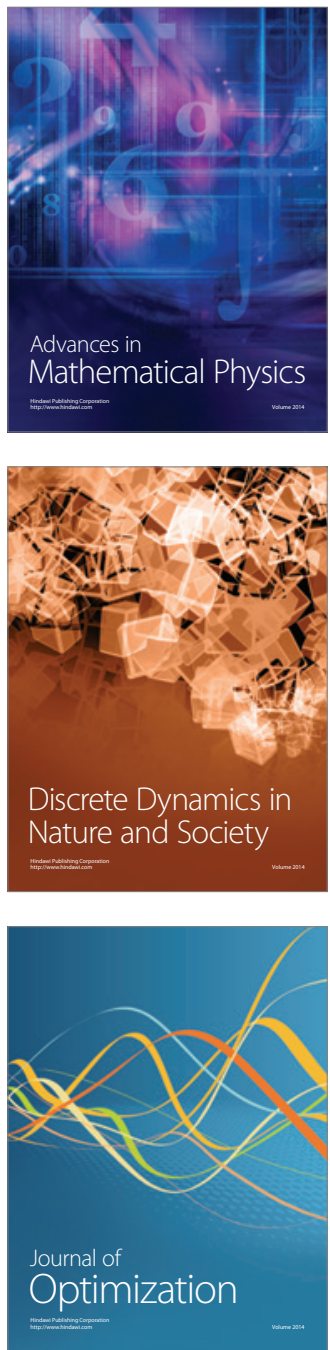